Neural response to emotional prosody

\title{
in schizophrenia and in bipolar affective disorder
}

\author{
RACHEL L. C. MITCHELL, REBECCA ELLIOTT, MARTIN BARRY, \\ ALAN CRUTTENDEN and PETER W. R. WOODRUFF
}

\begin{abstract}
Background Evidence suggests a reversal of the normal left-lateralised response to speech in schizophrenia.
\end{abstract}

\begin{abstract}
Aims To test the brain's response to emotional prosody in schizophrenia and bipolar disorder.
\end{abstract}

\section{Method BOLD contrast functional magnetic resonance imaging of subjects while they passively listened or attended to sentences that differed in emotional prosody.}

\begin{abstract}
Results Patients with schizophrenia exhibited normal right-lateralisation of the passive response to 'pure' emotional prosody and relative left-lateralisation of the response to unfiltered emotional prosody. When attending to emotional prosody, patients with schizophrenia activated the left insula more than healthy controls.When listening passively, patients with bipolar disorder demonstrated less activation of the bilateral superior temporal gyri in response to pure emotional prosody, and greater activation of the left superior temporal gyrus in response to unfiltered emotional prosody. In both passive experiments, the patient groups activated different lateral temporal lobe regions.
\end{abstract}

Conclusions Patients with schizophrenia and bipolar disorder may display some left-lateralisation of the normal right-lateralised temporal lobe response to emotional prosody.

Declaration of interest R.M. received a studentship from Neuraxis', and funding from the Neuroscience and Psychiatry Unit, University of Manchester.
Patients with schizophrenia demonstrate relatively greater activation of the right middle temporal gyrus, compared with the left, when listening to normal speech (Woodruff et al, 1997; Lennox et al, 2000; Sommer et al, 2001). The neural response to emotional prosody is normally seen in predominantly right hemisphere temporal lobe language regions (Heilman \& Gilmore, 1998; Buchanan et al, 2000; Mitchell et al, 2003), which raises the possibility that the right middle temporal gyrus 'hyper-responsivity' in patients with schizophrenia may be explained as an exaggerated functional response to emotional prosody. We thus tested two specific hypotheses: in patients with schizophrenia the cortical areas mediating the response to emotional prosody (particularly the middle temporal gyrus) are more sensitive to (and hence show a higher basal level of responsivity to) emotional prosody; and patients with schizophrenia over-attend to emotional prosody compared with healthy controls (and hence show increased topdown modulation of middle temporal gyrus activation in response to emotional prosody) (Woodruff et al, 1996).

\section{MATERIALS AND METHODS}

\section{Subjects}

Thirteen healthy controls were recruited from the staff and students of the University of Manchester. Twelve patients with schizophrenia (ICD-10 diagnosis; World Health Organization, 1993) and eleven patients with bipolar disorder (psychiatric controls, ICD-10 diagnosis; World Heath Organization, 1993) were recruited from out-patients attending Cromwell House Community Mental Health Centre (Salford

I. Formerly a contract research organisation devoted to the investigation of central nervous system drugs and their activity; now subsumed within Medeval (www.medeval.com). and Trafford NHS Trust) and the Psychiatry Department at Manchester Royal Infirmary (Central Manchester NHS Trust). The subjects' demographic statistics are summarised in Table 1 . Because gender and handedness each may influence lateralisation of the brain for language (Beaton, 1997), only male subjects who were right-handed (Annett, 1970) were studied. We excluded subjects with a history of hearing difficulties; those who did not speak English as their first language; any who were unable to consent; those who gave a self-report of neurological disorders; those with a history of alcohol or drug misuse, head injuries or long periods of unconsciousness; and those in whom magnetic resonance scanning was contraindicated. Additionally, controls who gave a self-report of psychiatric disorders were excluded. The study was conducted with full ethical approval from the Manchester University Ethics Committee and the local hospital ethics committees.

The symptomatology of patients with schizophrenia was assessed using the Scale for the Assessment of Positive Symptoms (SAPS; Andreasen, 1979a) and the Scale for the Assessment of Negative Symptoms (SANS; Andreasen, 1979b). Patients were not experiencing auditory hallucinations on the day of scanning as indicated by self-report. On the day of scanning, patients' delusion and hallucination scores were reassessed to determine whether there had been any changes in symptoms. Details of current medication and illness chronicity were obtained from patients' medical records. Medication levels in chlorpromazine equivalents were calculated for patients with schizophrenia (Taylor et al, 1999). Nine of the bipolar patients were receiving lithium carbonate or sodium valproate, four were receiving antipsychotic medication and five were being treated with antidepressants.

\section{Scanning paradigms}

A set of sentences were devised describing happy scenarios (e.g. She won the lottery jackpot) and sad scenarios (e.g. The dog had to be put down) that were approximately the same length and of a comparable style and format. The sentences were rated for perceived 'happiness' or 'sadness' by a group of control subjects $(n=20)$. The 60 sentences rated closest to the happy (0) and sad (10) ends of the 0 10 rating scale were selected for recording 
Table I Demographic and behavioural analyses according to subject group. Data presented as group mean (s.e.)

\begin{tabular}{|c|c|c|c|c|}
\hline & Healthy controls & $\begin{array}{l}\text { Patients with } \\
\text { schizophrenia }\end{array}$ & $\begin{array}{l}\text { Patients with } \\
\text { bipolar disorder }\end{array}$ & $\begin{array}{c}\text { Between-group } \\
\text { differences }\end{array}$ \\
\hline Age (years) & $32.2(3.6)$ & $45.7(2.7)$ & $42.8(1.8)$ & $\begin{array}{l}P=0.005 \\
C / S: P<0.01 \\
C / B P: P<0.05 \\
S / B P=N S\end{array}$ \\
\hline $\begin{array}{l}\text { Premorbid IQ } \\
\text { (NART') }\end{array}$ & $119.2(1.6)$ & III.8(3.7) & $116.9(2.9)$ & $P=0.166$ \\
\hline $\begin{array}{l}\text { Weekly alcohol } \\
\text { consumption (units) }\end{array}$ & $8.7(2.1)$ & $3.9(1.8)$ & $12.6(5.5)$ & $P=0.223$ \\
\hline Years education & $17.7(0.9)$ & $13.7(1.4)$ & I3.4 (I.I) & $\begin{array}{l}P=0.02 \\
C / S: P<0.05 \\
C / B P: P<0.05 \\
S / B P=N S\end{array}$ \\
\hline Original SAPS & N/A & $31.6(7.7)$ & N/A & N/A \\
\hline SANS & $\mathrm{N} / \mathrm{A}$ & 31.7 (3.7) & $\mathrm{N} / \mathrm{A}$ & $N / A$ \\
\hline $\begin{array}{l}\text { Delusion sub-scale } \\
\text { score on day of } \\
\text { scanning }\end{array}$ & N/A & $12.2(4.1)$ & $\mathrm{N} / \mathrm{A}$ & N/A \\
\hline $\begin{array}{l}\text { Hallucination sub- } \\
\text { scale score on day of } \\
\text { scanning }\end{array}$ & N/A & $6.0(1.9)$ & $\mathrm{N} / \mathrm{A}$ & $\mathrm{N} / \mathrm{A}$ \\
\hline $\begin{array}{l}\text { Performance } \\
\text { accuracy semantics }^{2}\end{array}$ & $61.0(3.2)$ & $57.1(4.2)$ & $62.9(5.3)$ & $P=0.631$ \\
\hline $\begin{array}{l}\text { Performance } \\
\text { accuracy prosody }{ }^{2}\end{array}$ & $70.0(5.1)$ & $60.7(1.8)$ & $68.1(4.6)$ & $P=0.206$ \\
\hline $\begin{array}{l}\text { Illness chronicity } \\
\text { (years) }\end{array}$ & $\mathrm{N} / \mathrm{A}$ & II.3 (I.4) & $8.2(1.4)$ & $P=0.145$ \\
\hline
\end{tabular}

On the whole, healthy controls, patients with schizophrenia and psychiatric controls were well-matched on the demographic and behavioural measures taken. $C / S$, difference between healthy controls and patients with schizophrenia; C/BP, difference between healthy controls and patients with bipolar disorder; S/BP, difference between patients with schizophrenia and patients with bipolar disorder; NS, non-significant difference; SAPS, Scale for the Assessment of Positive Symptoms; SANS, Scale for the Assessment of Negative Symptoms.

I. Nelson (1982).

2. Performance accuracy on the attentional task, as defined by the percentage of stimuli to which subjects responded correctly.

by an experienced phonetician (A.C.) in three styles of emotional intonation: happy, sad and neutral. Audiocassette recordings were then digitised at $22 \mathrm{kHz} / 16$ bits using an Apple Macintosh Centris 660 AV. A subset of sentences was bandpass filtered with a low-pass filter at $333 \mathrm{~Hz}$ to remove semantic information, thus creating 'pure' emotional prosodic stimuli. A second survey determined that subjects $(n=27)$ could reliably distinguish the emotion intoned in the filtered sentences. Paired $t$-tests revealed significant differences in emotional intonation rating (same scale as first survey) between sentences recorded in happy, sad and neutral styles of intonation (all $P<0.001)$.
Stimuli were presented as 37.8-s epochs consisting of eight contiguous stimuli, approximately $4.7 \mathrm{~s}$ in length, in an alternating $\mathrm{A} / \mathrm{B}$ block design. Each $\mathrm{A} / \mathrm{B}$ block was repeated three times. To dissect out the components of the response to emotional prosody, three separate scanning studies were performed. In the first experiment, passive listening to filtered emotional prosody was compared with the response to background scanner noise. In the second experiment, passive listening to unfiltered emotional prosodic sentences was compared with the response to background scanner noise. Pilot studies had suggested that comparing emotional prosody with neutral prosody was too subtle to achieve sufficient contrast. For the first two experiments, no active response was required. Subjects were asked to lie as still as possible, with their eyes closed, and listen passively. In these two passive experiments, sentences describing happy or sad scenarios were randomised to avoid habituation.

In the final experiment, subjects were presented with phrases depicting happy and sad scenarios and, when prompted, were instructed to attend to either semantic content or emotional intonation. In condition $\mathrm{A}$, subjects were asked to concentrate on what the speaker was saying, and if they thought that the speaker was describing a happy scenario they were to respond by squeezing the response bulb. In condition $\mathrm{B}$, they were required to ignore semantic content and concentrate on the speaker's emotional tone of voice, responding only when phrases were spoken in a happy tone of voice. Subjects performed this task in its entirety before entering the scanner to ensure that they were able to execute it properly. In this active experiment, we randomised statements where the emotion conveyed by intonation style was congruent with that conveyed by verbal content with those where the emotions conveyed by intonation and content were incongruent. In the passive listening experiments, the emotion provided by intonation and content was congruent throughout. The same series of sentences were used for all three experiments, i.e. verbal content remained the same. (All subjects performed all the scanning paradigms, in the order described above.)

\section{Image acquisition and analyses}

Auditory stimuli were presented using Macstim version 2.25 (David Darby, University of Melbourne) on a G3 Apple Macintosh PowerBook running operating system version 8.5 and played through a magnetic resonance-compatible patient MUSICBOX ${ }^{\mathrm{TM}}$ functional imaging sound system (Woodrow Premise, St Albans, UK). Before scanning, samples of the auditory stimuli were played to subjects positioned inside the scanner. If necessary, subject-specific volume adjustments were made to ensure that all stimuli were clearly heard. All functional magnetic resonance imaging (fMRI) experiments were performed on a Philips Gyroscan ACS NT 1.5 Tesla system (retrofitted with Powertrak 6000 gradients) operating at software level 6.1.2 (Hamburg, Germany). 
A T1-weighted turbo inversion recovery data set was acquired at 28 contiguous 3.5-mm slices acquired parallel to the body of the corpus callosum to achieve full brain coverage (echo time $=18 \mathrm{~ms}$, repetition time $=6850 \mathrm{~ms}$, in-plane resolution $=$ $0.89 \mathrm{~mm}$, field of view $=230 \mathrm{~mm}^{2}$ and acquisition matrix $=256 \times 256$ with one excitation). In the same orientation and position as for the structural scan (to capture all possible non-cerebellar haemodynamic responses), 72 T2*-weighted gradient echo, echo planar magnetic resonance images depicting BOLD contrast were acquired at each of 14 contiguous 7-mm thick slices. The single-shot imaging protocol comprised echo time $=50 \mathrm{~ms}$, repetition time $=3.1 \mathrm{~s}$, flip angle $=90^{\circ}$, echoplanar imaging factor $=63$, matrix $=$ $128 \times 128$, field of view $=230 \mathrm{~mm}^{2}$ and inplane resolution $=1.8 \mathrm{~mm}^{2}$. Total scan time was $3 \mathrm{~min} 47 \mathrm{~s}$ per individual experiment.

Data were analysed using SPM99 (Friston et al, 1995) implemented in MATLAB version 5.2 (The Mathworks Inc., MA, USA) and run on an Ultra 2 Creator 3D SUN workstation (Sun Microsystems, CA, USA) operating through Solaris version 3.5 (Sun Microsystems). Realignment, spatial normalisation and smoothing were performed prior to statistical analysis. Random effects analyses were carried out using a general linear model with a delayed boxcar waveform convolved with the haemodynamic response function. A high-pass filter was applied to the data to isolate the high-frequency effects of interest and to minimise physiological noise. Subject-specific low-frequency signal drift was removed by modelling with low-frequency sine and cosine waves (low-pass filter). Global flow effects were removed by proportional scaling.

Effects at each and every voxel were estimated and regionally specific effects were compared using linear contrasts. One mean contrast image was produced for each subject on the respective comparisons within the three experiments. For the group analyses, statistical inferences were based on the theory of random Gaussian fields, and a random effects model was applied. In the second-level analysis, two-sample $t$ tests were used to determine between-group differences. In view of the restricted activation differences predicted in the hypotheses (middle temporal gyrus), whole-brain correction was deemed too conservative. Thus, an uncorrected threshold of $P<0.001$ was adopted in regions of the middle temporal gyrus. Covariate analyses were performed to assess the relationship between chlorpromazine equivalent medication levels and functional activations in relation to all experiments (positive and negative correlations).

In summary, the interaction contrasts (i) to (vi) were performed in SPM for each of conditoins (a) to (d).

(a) When comparing passive listening to 'pure' filtered emotional prosody relative to rest... (b) When comparing passive listening to unfiltered emotional prosody relative to rest...

(c) When observing attention to emotional prosody (ignoring semantics)...

(d) When observing attention to semantics (ignoring emotional prosody)...

(i) . . . which regions were activated more in patients with schizophrenia than in healthy controls?

(ii) ... . which regions were activated less in patients with schizophrenia than in healthy controls?

Table 2 The neural response to emotional prosody in patients with schizophrenia

\begin{tabular}{|c|c|c|c|c|c|}
\hline Brain region & $\begin{array}{c}\text { Brodmann } \\
\text { area }\end{array}$ & Side & $\begin{array}{c}\text { Number of } \\
\text { voxels }\end{array}$ & $Z$ score & $\begin{array}{c}\text { Talairach } \\
\text { coordinates }\end{array}$ \\
\hline
\end{tabular}

Activated by 'pure' emotional prosody v. rest

\begin{tabular}{|c|c|c|c|c|c|c|}
\hline Middle temporal gyrus & 21 & $\mathbf{L}$ & 445 & 3.76 & $-63-21$ & -6 \\
\hline Middle temporal gyrus & 21 & $\mathbf{R}$ & 329 & 3.95 & $66-27$ & 0 \\
\hline Superior frontal gyrus & 6 & $\mathbf{R}$ & 14 & 3.56 & 15 & 63 \\
\hline Inferior parietal lobule & 40 & $\mathbf{R}$ & 20 & 3.21 & $48-30$ & 33 \\
\hline \multicolumn{7}{|c|}{ Activated by unfiltered emotional prosody v. rest } \\
\hline Superior temporal gyrus & 42 & $\mathbf{L}$ & 51 & 3.88 & $-42-27$ & 15 \\
\hline Superior temporal gyrus & 22 & $\mathrm{~L}$ & 293 & 3.46 & -57 & J \\
\hline Superior temporal gyrus & 22 & $\mathrm{~L}$ & 43 & 3.13 & $-48-45$ & 18 \\
\hline Middle temporal gyrus & 21 & $\mathbf{R}$ & 61 & 3.64 & $66-12$ & 0 \\
\hline Middle temporal gyrus & 21 & $\mathbf{R}$ & 153 & 3.54 & $54-48$ & 0 \\
\hline Hippocampus & N/A & $\mathrm{L}$ & 26 & 3.72 & $-36-24$ & -12 \\
\hline Inferior frontal gyrus & 44 & $\mathbf{R}$ & 28 & 3.09 & 48 & 30 \\
\hline
\end{tabular}

Inferior frontal gyrus

44

R 28

3.09

$48 \quad 9 \quad 30$

Activated by attention to semantics (ignoring emotional prosody)

$\begin{array}{llllllll}\text { Inferior parietal lobule } & 40 & R & 26 & 3.39 & 66 & -39 & 33\end{array}$

Activated by attention to emotional prosody (ignoring semantics)

\begin{tabular}{|c|c|c|c|c|c|c|c|}
\hline Superior temporal gyrus & 38 & $\mathbf{R}$ & 12 & 3.48 & 45 & 12 & -15 \\
\hline Insula & N/A & $\mathbf{L}$ & 1009 & 4.60 & -27 & -18 & 21 \\
\hline Insula & $\mathrm{N} / \mathrm{A}$ & $\mathbf{R}$ & 35 & 3.61 & 36 & -18 & 3 \\
\hline Superior frontal gyrus & 8 & $\mathbf{R}$ & 20 & 3.16 & 15 & 30 & 54 \\
\hline Middle frontal gyrus & 9 & $\mathbf{L}$ & 75 & 3.26 & -30 & 15 & 36 \\
\hline Middle frontal gyrus & 46 & $\mathbf{R}$ & 10 & 3.47 & 51 & 30 & 18 \\
\hline Precentral gyrus & 4 & $\mathbf{R}$ & 62 & 3.51 & 21 & -24 & 63 \\
\hline Superior parietal lobule & 7 & $\mathbf{R}$ & 82 & 3.57 & 15 & -60 & 60 \\
\hline Inferior parietal lobule & 40 & $\mathbf{R}$ & 31 & 3.17 & 39 & -45 & 57 \\
\hline Post-central gyrus & 40 & $\mathrm{~L}$ & 143 & 4.57 & -57 & -27 & 24 \\
\hline Fusiform gyrus & 37 & $\mathrm{~L}$ & 25 & 3.24 & -33 & -45 & -10 \\
\hline Lingual gyrus & 19 & $\mathbf{R}$ & 12 & 3.20 & 9 & -69 & -3 \\
\hline Pulvinar & $\mathrm{N} / \mathrm{A}$ & $\mathbf{R}$ & 21 & 3.20 & 21 & -27 & \\
\hline Putamen & N/A & $\mathbf{L}$ & 40 & 3.23 & -24 & 15 & \\
\hline
\end{tabular}

Regions of statistically significant activation in patients with schizophrenia during their passive and active response to emotional prosody. Areas of statistically significant difference are summarised by brain region and by corresponding Brodmann area in columns I and 2, respectively. The third column indicates the hemisphere in which the 'activation' occurred ( $L=$ left, $R=$ right). The fourth column summarises the number of 'active' voxels and indicates the spatial extent of the active region, whereas $Z$ scores in the fifth column indicate the intensity of activity. Regions of statistically significant difference between experimental conditions were significant to $P<0.001$. Coordinates are given for the stereotacic space of Talairach \& Tournoux (1988) in the last column. 
Table 3 The neural response to emotional prosody in patients with bipolar disorder

\begin{tabular}{|c|c|c|c|c|c|c|c|}
\hline Brain region & $\begin{array}{l}\text { Brodmann } \\
\text { area }\end{array}$ & Side & $\begin{array}{l}\text { Number } \\
\text { of voxels }\end{array}$ & Z score & \multicolumn{3}{|c|}{$\begin{array}{c}\text { Talairach } \\
\text { coordinates }\end{array}$} \\
\hline \multicolumn{8}{|c|}{ Activated by 'pure'emotional prosody v. rest } \\
\hline Superior temporal gyrus & 38 & $\mathbf{R}$ & 27 & 3.47 & 36 & 6 & -27 \\
\hline Middle temporal gyrus & 21 & $\mathbf{L}$ & 1090 & 5.20 & -63 & -12 & -3 \\
\hline Middle temporal gyrus & $\mathbf{2 l}$ & $\mathbf{R}$ & 665 & 5.03 & 54 & -12 & -3 \\
\hline Inferior frontal gyrus & 45 & $\mathbf{L}$ & 38 & 3.19 & -54 & 24 & 18 \\
\hline \multicolumn{8}{|c|}{ Regions activated by unfiltered emotional prosody v. rest } \\
\hline Middle temporal gyrus & 21 & $\mathbf{L}$ & 792 & 4.43 & -60 & -15 & -3 \\
\hline Middle temporal gyrus & 21 & $\mathbf{R}$ & 569 & 4.13 & 63 & -15 & -6 \\
\hline Inferior frontal gyrus & 44 & L & 32 & 3.30 & -36 & 15 & 12 \\
\hline
\end{tabular}

Regions of statistically significant activation in patients with bipolar disorder during their passive response to emotional prosody. Areas of statistically significant difference are summarised by brain region and by Brodmann area in columns $I$ and 2 , respectively. The third column indicates the hemisphere of 'activation' ( $L=$ left, $R=$ right), the fourth column summarises the spatial extent of the active region and the $Z$ scores in the fifth column indicate the intensity of activity. Regions of statistically significant difference between experimental conditions were significant to $P<0.001$. Coordinates are given for the stereotactic space of Talairach \& Tournoux (1988) in the last column. Results that survive correction for multiple comparisons at $P \leqslant 0.05$ are in bold type. There were no statistically significant areas of activation during attention to semantics (ignoring emotional prosody) or during attention to emotional prosody (ignoring semantics).

Table 4 Differences between healthy controls and patients with schizophrenia in the passive neural response to filtered ('pure') and unfiltered emotional prosody

\begin{tabular}{|c|c|c|c|c|c|}
\hline Brain region & $\begin{array}{c}\text { Brodmann } \\
\text { area }\end{array}$ & Side & $\begin{array}{c}\text { Number of } \\
\text { voxels }\end{array}$ & $Z$ score & $\begin{array}{c}\text { Talairach } \\
\text { coordinates }\end{array}$ \\
\hline
\end{tabular}

Activated by patients with schizophrenia more than healthy controls in response to 'pure'emotional prosody

$\begin{array}{lrrrrrrr}\text { Inferior parietal lobule } & 40 & \mathrm{~L} & 37 & 3.25 & -60 & -33 & 42 \\ \text { Precentral gyrus } & 4 & \mathrm{R} & 223 & 4.02 & 51 & -3 & 18\end{array}$

Activated by patients with schizophrenia more than healthy controls in response to unfiltered emotional prosody

\begin{tabular}{|c|c|c|c|c|c|}
\hline Middle temporal gyrus & 21 & $\mathbf{L}$ & 43 & 3.15 & $\begin{array}{lll}-48 & -54 & -9\end{array}$ \\
\hline Parahippocampal gyrus & 36 & $\mathbf{L}$ & 23 & 3.60 & $-36-24-15$ \\
\hline Precentral gyrus & 6 & $\mathbf{R}$ & 57 & 3.28 & 39 \\
\hline
\end{tabular}

Activated by patients with schizophrenia less than healthy controls in response to unfiltered emotional prosody

\begin{tabular}{|c|c|c|c|c|c|}
\hline Superior temporal gyrus & 42 & L & 66 & 3.30 & $-48-21$ \\
\hline Precuneus & 19 & $\mathbf{R}$ & 67 & 3.20 & $3-69$ \\
\hline
\end{tabular}

Areas of statistically significant difference are summarised by brain region and by corresponding Brodmann area in columns $I$ and 2 , respectively. The third column indicates the hemisphere in which the 'activation' occurred $(L=l$ left, $\mathrm{R}=$ right). The fourth column summarises the number of active voxels and indicates the spatial extent of the active region, whereas $Z$ scores in the fifth column indicate the intensity of activity. Group differences in activations were significant to $P<0.001$, uncorrected. Coordinates are given for the stereotactic space of Talairach \& Tournoux (1988) in the last column. Within the table, data include regions of greater and lesser activity. In response to 'pure' emotional prosody, no regions were activated significantly more in healthy controls than in patients with schizophrenia.

(iii) ... which regions were activated more in patients with schizophrenia than in patients with bipolar disorder?

(iv) ... which regions were activated less in patients with schizophrenia than in patients with bipolar disorder? (v) ... which regions were activated more in healthy controls than in patients with bipolar disorder?

(vi) ... which regions were activated less in healthy controls than in patients with bipolar disorder?

\section{RESULTS}

Statistical analyses of the demographic and behavioural data are summarised in Table 1. Here we summarise the temporal lobe activations that were significant at a threshold of $P<0.001$. Supra-threshold activations about which we did not have an $a$ priori hypothesis are summarised in Tables 2-6. Tables 2 and 3 summarise the withingroup contrasts for both patient groups. A full report on the neural response to emotional prosody in our healthy control subjects can be found in Mitchell et al (2003). However, in light of our hypotheses, it was the between-group comparisons that were of primary interest (Tables 4, 5 and 6) and it is the betweengroup differences that are the focus of the discussion that follows.

\section{Scanning session I: filtered emotional prosody v. rest}

Consistent with our hypothesis, patients with schizophrenia activated the right anterior middle temporal gyrus to a greater extent than patients with bipolar disorder in response to passive listening to filtered emotional prosody. However, patients with bipolar disorder demonstrated greater activation than those with schizophrenia on the same task in another region of right middle temporal gyrus, posterior to that shown to be more responsive in patients with schizophrenia.

\section{Scanning session 2: emotional prosody in normal speech $v$. rest}

In patients with schizophrenia relative to healthy controls, contrary to our prediction of a greater right middle temporal gyrus response to emotional prosody in schizophrenia, emotional prosody in normal speech induced greater activation of the left middle temporal gyrus. On the other hand, healthy controls did activate the left superior temporal gyrus to a greater extent than did patients with schizophrenia.

Patients with schizophrenia also demonstrated relatively greater activation bilaterally in the superior temporal gyrus and the left middle temporal gyrus compared with patients with bipolar disorder. However, patients with bipolar disorder demonstrated relatively greater activation of lateral and anterior portions of the superior temporal gyrus than did patients with schizophrenia. 


\section{Scanning session 3 (not tabulated)}

Attention to semantics (ignoring emotional prosody)

No brain regions activated to a greater extent in patients with schizophrenia than in controls. Healthy controls activated the left middle frontal (Brodmann area 46, 197 voxels, $Z$ score $3.52, x=-36, y=33$, $z=21)$ and right inferior frontal gyri (Brodmann area 46,19 voxels, $Z$ score $3.66, x=48, y=30, z=12$ ) to a greater extent than patients with schizophrenia.

\section{Attention to emotional prosody (ignoring semantics)}

When attending to emotional prosody, patients with schizophrenia activated the left insula to a greater extent than did healthy controls ( 180 voxels, $Z$ score 3.71 , $x=-33, y=-18, z=15)$ but they did not demonstrate increased activation of the right middle temporal gyrus compared with healthy controls. No brain regions were activated to a greater extent in healthy controls than in patients with schizophrenia.

As the chlorpromazine equivalent medication levels in patients with schizophrenia attending to emotional prosody increased, so did the neural activity in the left superior temporal gyrus, insula and superior frontal gyrus. However, there was no overlap between areas in which activity was linked to medication levels and areas recruited by patients to attend to emotional prosody.

In conjunction with analyses of the main effects of the subject group described above, further statistical analyses were performed to assess between-group differences in the relative laterality of response to emotional prosody. For each of the three experiments, a general linear model analysis of peak lateral temporal lobe activation $Z$ scores was performed to examine interactions between subject group and the hemisphere in which lateral temporal lobe activation was greatest. The region of interest was defined as including activations in the superior and middle temporal gyri. The results of these analyses are summarised in Table 7.

\section{DISCUSSION}

\section{Main results}

(a) Patients with schizophrenia demonstrated some reversal of the normal right-lateralised temporal lobe response
Table 5 Differences between patients with schizophrenia and patients with bipolar disorder in the passive neural response to emotional prosody

Brain region Brodmann area Side Number of voxels $Z$ score Talairach coordinates

Activated by patients with schizophrenia more than patients with bipolar disorder in response to 'pure' emotional prosody

Middle temporal gyrus

Middle temporal gyrus

Cingulate gyrus

$\begin{array}{lll}21 & R & 10 \\ 21 & R & 19 \\ 24 & R & 37 \\ 40 & \text { L } & 56\end{array}$

$\begin{array}{lrrrr}10 & 3.46 & 36 & 3 & -27 \\ 19 & 3.20 & 60 & -30 & -9 \\ 37 & 4.03 & 3 & 3 & 30 \\ 56 & 3.62 & -48 & -45 & 27\end{array}$

Supramarginal gyrus

40

56

$\begin{array}{llll}3.62 & -48 & -45 & 27\end{array}$

Activated by patients with schizophrenia less than patients with bipolar disorder in response to 'pure' emotional prosody

$\begin{array}{llllllll}\text { Middle temporal gyrus } & 39 & R & 42 & 3.38 & 36 & -60 & 18\end{array}$

Activated by patients with schizophrenia more than patients with bipolar disorder in response to unfiltered emotional prosody

$\begin{array}{llllllll}\text { Superior temporal gyrus } & 38 & \mathrm{~L} & 13 & 3.34 & -30 & 9 & -24\end{array}$

$\begin{array}{llllllll}\text { Superior temporal gyrus } & 22 & R & 37 & 3.33 & 57 & -27 & 3\end{array}$

$\begin{array}{lllllllll}\text { Middle temporal gyrus } & 21 & \mathrm{~L} & 27 & 3.32 & -57 & -48 & 3\end{array}$

Activated by patients with schizophrenia less than patients with bipolar disorder in response to unfiltered emotional prosody

$\begin{array}{llllllll}\text { Superior temporal gyrus } & 22 & \mathrm{~L} & 22 & 3.41 & -57 & 0 & 3\end{array}$

$\begin{array}{llllllll}\text { Parahippocampal gyrus } & 27 & R & 30 & 3.64 & 21 & -33 & -6\end{array}$

$\begin{array}{llllllll}\text { Middle frontal gyrus } & 6 & \mathrm{~L} & 28 & 3.24 & -33 & 0 & 45\end{array}$

$\begin{array}{llllllll}\text { Precentral gyrus } & 6 & R & \text { II } & 3.12 & 42 & -6 & 42\end{array}$

Areas of statistically significant difference are summarised by brain region and by corresponding Brodmann area in columns $I$ and 2 , respectively. The third column indicates the hemisphere in which the 'activation' occurred $(L=l$ left, $\mathrm{R}=$ right) the fourth column indicates the spatial extent of the active region and the fifth column indicates the intensity of activity. Group differences in activations were significant to $P<0.00 \mathrm{I}$, uncorrected. Coordinates are given for the stereotactic space of Talairach \& Tournoux (1988) in the last column. Within the table, data include regions of greater and lesser activity.

Table 6 Differences between healthy controls and patients with bipolar disorder in the passive neural response to emotional prosody

Brain region Brodmann area Side Number of voxels $Z$ score Talairach coordinates

Activated by healthy controls more than patients with bipolar disorder in response to 'pure' emotional prosody

$\begin{array}{lccccrrr}\text { Superior temporal gyrus } & 42 & \mathrm{R} & 80 & 3.41 & 57 & -27 & 15 \\ \text { Superior temporal gyrus } & 22 & \mathrm{~L} & 57 & 3.24 & -5 \mathrm{I} & -45 & 12 \\ \text { Amygdala } & \mathrm{N} / \mathrm{A} & \mathrm{R} & 84 & 3.86 & 21 & 3 & -18 \\ \text { Uncus } & 28 & \mathrm{~L} & 55 & 3.72 & -27 & 0 & -24 \\ \text { Inferior frontal gyrus } & 47 & \mathrm{R} & 23 & 3.12 & 30 & 21 & -21 \\ \text { Precentral gyrus } & 6 & \mathrm{R} & 58 & 3.27 & 45 & 3 & 48 \\ \text { Post-central gyrus } & 40 & \mathrm{~L} & 32 & 3.17 & -57 & -27 & 51\end{array}$

Activated by healthy controls more than patients with bipolar disorder in response to unfiltered emotional prosody

$\begin{array}{lccccccc}\text { Hippocampus } & \text { N/A } & R & 19 & 3.51 & 24 & -33 & -6 \\ \text { Supramarginal gyrus } & 40 & R & 134 & 4.03 & 45 & -48 & 36\end{array}$

Activated by healthy controls less than patients with bipolar disorder in response to unfiltered emotional prosody

Uncus

28

L

35

$3.99-33 \quad 3-2$

Areas of statistically significant difference are summarised by brain region and by corresponding Brodmann area in columns $I$ and 2, respectively. The third column indicates the hemisphere in which the 'activation' occurred $(L=$ left, $R=$ right), the fourth column indicates the spatial extent of the active region and the fifith column indicates the intensity of activity. Group differences in activations were significant to $P<0.001$, uncorrected. Coordinates are given for the stereotactic space of Talairach \& Tournoux (1988) in the last column. Within the table, data include regions of greater and lesser activity. In response to 'pure' emotional prosody, no regions were activated significantly more in patients with bipolar disorder than in healthy controls. 
Table 7 Temporal lobe interactions between subject group and hemisphere

\begin{tabular}{|c|c|c|c|c|c|c|}
\hline Contrast & $\begin{array}{l}\text { Subject } \\
\text { group }\end{array}$ & $\begin{array}{c}\text { Mean peak left lateral } \\
\text { temporal lobe } \\
Z \text { score }\end{array}$ & $\begin{array}{c}\text { Mean peak right lateral } \\
\text { temporal lobe } \\
Z \text { score }\end{array}$ & $\begin{array}{c}F \text { value of subject } \\
\text { group by hemisphere } \\
\text { GLM analysis }\end{array}$ & $\begin{array}{c}\text { GLM significance } \\
\text { level }\end{array}$ & $\begin{array}{c}\text { Post hoc analyses of } \\
\text { significant GLM } \\
\text { results }\end{array}$ \\
\hline Pure emotional prosody & $\mathrm{HC}$ & $3.21(0.110)$ & $3.59(0.222)$ & 2.240 & 0.122 & \\
\hline \multirow[t]{2}{*}{ v. rest } & $\mathrm{S}$ & $4.83(0.273)$ & $4.47(0.314)$ & & & \\
\hline & BP & $4.74(0.222)$ & $4.50(0.37 I)$ & & & \\
\hline Unfiltered emotional & $\mathrm{HC}$ & $4.90(0.327)$ & $5.07(0.30 \mathrm{I})$ & 3.874 & 0.031 & $H C: R>L$ \\
\hline \multirow[t]{2}{*}{ prosody $v$. rest } & $\mathrm{S}$ & $5.30(0.256)$ & $5.03(0.364)$ & & & $S: L>R$ \\
\hline & BP & $5.47(0.427)$ & $4.20(0.426)$ & & & \\
\hline Attention to semantics & $\mathrm{HC}$ & $3.95(0.283)$ & $3.74(0.223)$ & 0.242 & 0.787 & \\
\hline (ignoring emotional & $\mathrm{S}$ & $3.57(0.316)$ & $3.51(0.257)$ & & & \\
\hline prosody) & $\mathrm{BP}$ & $3.46(0.223)$ & $3.43(0.139)$ & & & \\
\hline Attention to emotional & $\mathrm{HC}$ & $3.54(0.197)$ & $3.53(0.186)$ & 0.459 & 0.636 & \\
\hline prosody & $\mathrm{S}$ & $3.28(0.096)$ & $3.36(0.100)$ & & & \\
\hline (ignoring semantics) & $\mathrm{BP}$ & $3.56(0.324)$ & $3.31(0.165)$ & & & \\
\hline
\end{tabular}

A general linear model (GLM) analysis was performed to determine subject group ( $\mathrm{HC}$, healthy control; S, schizophrenia; $\mathrm{BP}$, bipolar disorder) by hemisphere $Z$ score interactions in the lateral temporal lobe, according to experimental contrast. Post hoc analyses revealed that, when listening passively to unfiltered emotional prosody, patients with schizophrenia showed significantly greater activation of the left $(\mathrm{L})$ temporal lobe than of the right $(\mathrm{R})$ and healthy controls demonstrated significantly greater activation of the right temporal lobe than of the left.

to emotional prosody, particularly in response to unfiltered emotional prosody.

(b) Patients with bipolar disorder also displayed some evidence of a reversal of the normal temporal lobe response to emotional prosody.

(c) When actively attending to emotional prosody, patients with schizophrenia activated the left insula to a greater extent than did healthy controls.

(d) When listening to pure emotional prosody, patients with bipolar disorder activated the amygdala, bilateral superior temporal gyrus and right inferior frontal gyrus less than did controls.

\section{Key abnormalities in patients with schizophrenia}

We hypothesised that for both passive and active listening to emotional prosody patients with schizophrenia would demonstrate greater activation of the right middle temporal gyrus than healthy control subjects. This hypothesis presupposed that, like healthy controls, patients with schizophrenia would activate the right middle temporal gyrus to process emotional prosody, albeit to a greater degree. Contrary to our hypotheses, however, patients with schizophrenia demonstrated some reversal of the normal right-lateralised temporal lobe response to emotional prosody (i.e. left more than right). In response to both pure emotional prosody and emotional prosody in normal speech, the within-group $Z$ scores (Tables 2 and 3 ) suggested that, compared with rest, healthy controls activated the right lateral temporal lobe more than the left, and that patients with schizophrenia activated the left temporal lobe more than the right. In response to unfiltered emotional prosody, the between-group comparisons demonstrated that patients with schizophrenia activated the left middle temporal gyrus significantly more than did healthy controls, and post hoc analyses established that there was a highly significant interaction between subject group and hemisphere, such that patients with schizophrenia showed greater activation of the left lateral temporal lobe than of the right, and healthy controls demonstrated greater activation of the right lateral temporal lobe than of the left (see Table 7).

Previous studies suggest that there may be some reversal of the normal leftlateralised temporal lobe response to semantics in patients with schizophrenia (Woodruff et al, 1997). This previously demonstrated right lateralisation of response to semantics in schizophrenia could feasibly be accompanied by a left lateralisation of the response to emotional prosody. Indeed, such a double reversal of dominance was described by Joseph (1986) in his post-surgical investigation of a patient who had undergone neurosurgery to alleviate early-onset left-hemisphere epilepsy. That case study supports the idea that the left hemisphere could 'take over' functions normally subserved by the right hemisphere if the right hemisphere is damaged sufficiently and early enough in brain development. However, our abnormal lateralisation findings are confined to emotional prosody. Our observation that emotional prosody is sometimes left lateralised in schizophrenia means that an excessive cortical response to emotional prosody in schizophrenia is unlikely to explain the right-sided bias in their cortical response to external speech (Woodruff et al, 1997). Furthermore, the similarity of our observation in patients with schizophrenia to that observed in individuals with bipolar disorder suggests that factors that lead to this right-left reversal or failure of normal lateralisation are common to some general 'psychosis factor', not necessarily specific to schizophrenia alone. Despite this similarity, patients with schizophrenia and bipolar disorder activated different temporal lobe regions from each other in response to filtered and unfiltered emotional prosody. The data suggest that, in our tasks, schizophrenia and bipolar disorder are associated with functional abnormalities in different regions of the middle and superior temporal gyri (Table 5). It is possible that the two diseases may differentially affect temporal lobe function, perhaps reflecting observed differences in language (Docherty et al, 
1996; Thomas et al, 1996) and auditory event-related potential disturbances (Souza et al, 1995) between these two patient groups.

A similarly noteworthy result arose when attention to emotional prosody was compared with attention to semantics in patients with schizophrenia relative to healthy controls. In this active condition, patients with schizophrenia activated the left insula to a greater extent than did healthy controls. It has been reported previously (Shapleske et al, 2002) that in patients with schizophrenia the insula is reduced in size compared with healthy controls. Top-down attentional modulation is a means by which directing attention towards a stimulus in one sensory modality enhances cortical activation in sensory cortex of the same modality (Woodruff $e t$ al 1996). Together, these findings could indicate that in patients with schizophrenia the insula is hyper-responsive to attentional modulation of the response to speech that contains emotional prosody. In this context, hyper-responsivity of the insula could reflect a reduction of the insula inhibition that normally occurs during self-regulation of emotional response (Beauregard et al, 2001), as part of the affect regulation difficulties (Shaw et al, 1999) or fronto-temporal disinhibition that characterise the disorder (Friston et al, 1992).

\section{Other abnormalities in patients with bipolar disorder}

Compared with healthy controls, patients with bipolar disorder listening to pure emotional prosody demonstrated significantly less activation of the amygdala, uncus, bilateral superior temporal gyrus and right inferior frontal gyrus. Previous neuroimaging studies have demonstrated that emotional prosody recognition normally activates the right prefrontal cortex (George et al, 1996; Buchanan et al, 2000) and the right anterior auditory cortex (Buchanan et al, 2000; Mitchell et al, 2003). We speculate that the relative lack of right superior temporal and inferior frontal gyrus activity in patients with bipolar disorder could indicate that this patient group has a reduced (neural) capacity to process the emotional prosody tested in our study, or that this capacity is under-utilised. Our finding of significantly decreased amygdala activation in patients with bipolar disorder compared with healthy controls listening to pure emotional prosody is consistent with structural

\section{CLINICAL IMPLICATIONS}

- Owing to the importance of emotional interpretation for successful social integration, further attention should be paid to the role of emotional prosodic processing dysfunction in the communicative abilities of people with schizophrenia.

- The role of emotional prosody in misinterpretation of statements or reinforcement of paranoid activity as occurs in delusions should be considered in more depth.

- Our findings may increase understanding of the functional implications of abnormal cerebral lateralisation. The abnormal lateralisation of emotional prosodic processing in schizophrenia could predispose patients to symptoms such as auditory hallucinations and thought disorder.

\section{LIMITATIONS}

- In common with functional imaging studies in general, this study did not specifically address the impact of any structural abnormalities on functional abnormalities.

Because there was only a limited measure of output during passive listening to emotional prosody (debriefing), we cannot be specific about the additional cognitive operations, perhaps unrelated to the task, that patients may have been engaging while in the scanner.

Although the covariate analyses suggested a lack of association between the response to emotional prosody and medication levels, the correlational approach cannot prove or rule out whether medication affects the emotional prosodic processing in schizophrenia.

RACHEL L. C. MITCHELL, PhD, School of Psychology, University of Reading, and Neuroscience and Emotion Section, Institute of Psychiatry, London; REBECCA ELLIOTT, PhD, Neuroscience and Psychiatry Unit, University of Manchester; MARTIN BARRY, MPhil, ALAN CRUTTENDEN, PhD, Department of Linguistics, University of Manchester; PETER W. R. WOODRUFF, PhD, MRCP, MRCPsych, SCANLab, Academic Department of Psychiatry, University of Sheffield

Correspondence: Dr Rachel Mitchell, School of Psychology, University of Reading,Whiteknights Road, Reading, Berkshire RG6 6AL, UK

(First received II April 2003, final revision 30 July 2003, accepted 29 October 2003)

amygdala abnormalities well documented in bipolar disorder (Strakowski et al, 1999; Altshuler et al, 2000). The existence of functional amygdala abnormalities in bipolar disorder in response to emotional stimuli has been demonstrated already, in facial emotion discrimination tasks (Kennedy et al, 1997; Yurgelun-Todd et al, 2000).

\section{General issues}

Because patients did not demonstrate a performance deficit, the patient-control differences in functional activations can be ascribed to neural processes in schizophrenia itself rather than to behavioural impairment alone. Furthermore, contrary to recent hypotheses (Heinrichs \& Zakzanis, 1998; Bilder et al, 2000), patients with schizophrenia did not demonstrate global attenuation of brain activity, because when processing emotional prosody they activated several brain regions not activated by healthy controls. In this respect, it is important to note that, in response to emotional prosody, patients with schizophrenia actually activated some brain regions more than did healthy controls, suggesting that when performing certain 
processing tasks this patient group has the capacity to recruit brain regions and cognitions that healthy controls cannot.

\section{Further work}

In a future study, it would be interesting to examine whether, like prosodic comprehension, prosodic expression is mediated abnormally by patients with schizophrenia. It is not clear from the literature whether patients with schizophrenia have a tendency to be abnormally right lateralised for the production of speech as well as its comprehension.

Patients with bipolar affective disorder may experience several different phases of illness. Thus, it is likely that a patient's cognitive dysfunctions and neural response to emotional prosody also may vary in phases. To support the hypothesis that emotional dysfunctions are related to emotional prosodic abnormalities in patients with bipolar disorder, it would be informative to determine whether this patient group also responds abnormally to non-emotional or linguistic prosody.

\section{REFERENCES}

Altshuler, L. L., Bartzokis, G., Grieder, T., et al (2000) An MRI study of temporal lobe structures in men with bipolar disorder or schizophrenia. Biological Psychiatry, 48, 147-162.

Andreasen, N. C. (1979a) Scale for the Assessment of Positive Symptoms. lowa, IA: University of lowa.

Andreasen, N. C. (1979b) Scale for the Assessment of Negative Symptoms. lowa, IA: University of lowa.

Annett, M. (1970) A classification of hand preference by association analysis. British Journal of Psychology, $\mathbf{6 1}$, 303-321.

Beaton, A. A. (1997) The relation of planum temporale asymmetry and morphology of the corpus callosum to handedness, gender, and dyslexia: a review of the evidence. Brain and Language, 60, 255-322.

Beauregard, M., Levesque, J. \& Bourgouin, P. (200I) Neural correlates of conscious self-regulation of emotion. Journal of Neuroscience, 2I, I-6.

Bilder, R. M., Goldman, R. S., Robinson, D., et al (2000) Neuropsychology of first-episode schizophrenia: initial characterization and clinical correlates. American Journal of Psychiatry, 157, 549-559.

Buchanan, T.W., Lutz, K., Mirzazade, S., et al (2000) Recognition of emotional prosody and verbal components of spoken language: an fMRI study. Brain Research. Cognitive Brain Research, 9, 227-238.

Docherty, N. M., DeRosa, M. \& Andreasen, N. C. (1996) Communication disturbances in schizophrenia and mania. Archives of General Psychiatry, 53, 358-364.

Friston, K. J., Liddle, P. F., Frith, C. D., et al (1992) The left medial temporal region and schizophrenia: a PET study. Brain, II5, 367-382.

Friston, F. J., Holmes, A. P., Worsley, K. J., et al (1995) Statistical parametric mapping in functional imaging: a general approach. Human Brain Mapping, 2, 189-210.

George, M. S., Parekh, P. I., Rosinsky, N., et al (1996) Understanding emotional prosody activates right hemisphere regions. Archives of Neurology, 53, 665-670.

Heilman, K. M. \& Gilmore, R. L. (1998) Cortica influences in emotion. Journal of Clinical Neurophysiology, I5, 409-423.

\section{Heinrichs, R.W. \& Zakzanis, K. K. (1998)}

Neurocognitive deficit in schizophrenia: a quantitative review of the evidence. Neuropsychology, 12, 426-445.

Joseph, R. (1986) Reversal of cerebral dominance for language and emotion in corpus callostomy patient. Journal of Neurology, Neurosurgery and Psychiatry, 52, 736-74l.

Kennedy, S. H., Javanmard, M. \& Vaccarino, F. J. (1997) A review of functional neuroimaging in mood disorders: positron emission tomography and depression. Canadian Journal of Psychiatry, 42, 467-475.

Lennox, B. R., Park, S. B., Medley, l., et al (2000) The functional anatomy of auditory hallucinations in schizophrenia. Psychiatry Research, 100, 13-20.

Mitchell, R. L. C., Elliott, R., Barry, M., et al (2003) The neural response to emotional prosody, as revealed by functional magnetic resonance imaging. Neuropsychologia, 4I, |4I0-1421.
Nelson, H. E. (1982) National Adult Reading Test (NART): Test Manual.Windsor: NFER-Nelson.

Shapleske, J., Rossell, S. L., Chitnis, X. A., et al (2002) A computational morphometric MRI study of schizophrenia: effects of hallucinations. Cerebral Cortex, |2, |33|-|34|.

Shaw, R. J., Dong, M., Lim, K. O., et al (1999) The relationship between affect expression and affect recognition in schizophrenia. Schizophrenia Research, 37, 245-250.

Sommer, I. E. C., Ramsey, N. F. \& Kahn, R. S. (200I) Language lateralization in schizophrenia, an fMRI study. Schizophrenia Research, 52, 57-67.

Souza, V. B., Muir, W. J., Walker, M. T., et al (1995) Auditory P300 event-related potentials and neuropsychological performance in schizophrenia and bipolar affective disorder. Biological Psychiatry, 37. 300-310.

Strakowski, S. M., DelBello, M. P., Sax, K.W., et al (1999) Brain magnetic resonance imaging of structura abnormalities in bipolar disorder. Archives of General Psychiatry, 56, 254-260.

Talairach, J. \& Tournoux, P. (1988) Co-Planar Stereotaxic Atlas of the Human Brain. New York: Thieme Medical.

Taylor, D., McConnell, D., McConnell, H., et al (1999) The Bethlem and Maudsley NHS Trust Prescribing Guidelines 1999 (5th edn). London: Martin Dunitz.

Thomas, P., Kearney, G., Napier, E., et al (1996) Speech and language in first onset psychosis. Differences between people with schizophrenia, mania, and controls. British Journal of Psychiatry, 168, 337-343.

Woodruff, P. W., Benson, R. R., Bandettini, P. A., et al (1996) Modulation of auditory and visual cortex by selective attention is modality-dependent. NeuroReport, 7. 1909-1913.

Woodruff, P. W., Wright, I. C., Bullmore, E.T., et al (1997) Auditory hallucinations and the temporal cortical response to speech in schizophrenia: a functional magnetic resonance imaging study. American Journal of Psychiatry, 154, 1676-1682.

World Health Organization (1993) The ICD-10 Classification of Mental and Behavioural Disorders: Diagnostic Criteria for Research, pp. 64-69. Geneva: WHO.

Yurgelun-Todd, D. A., Gruber, S. A., Kanayama, G. et al (2000) fMRI during affect discrimination in bipolar affective disorder. Bipolar Disorder, 2, 237-248. 